ORIGINAL ARTICLE

\title{
Mortality of employees of a perfluorooctanesulphonyl fluoride manufacturing facility
}

\author{
B H Alexander, G W Olsen, J M Burris, J H Mandel, J S Mandel
}

Occup Environ Med 2003;60:722-729

See end of article for authors' affiliations

.....................

Correspondence to:

Dr B H Alexander,

University of Minnesota,

School of Public Health,

Division of Environmental

and Occupational Health

MMC 807 Mayo Building,

420 Delaware St, SE

Minneapolis, MN 55409,

USA; balex@umn.edu

Accepted

23 December 2002

\begin{abstract}
Aim: To evaluate the mortality experience of a cohort of employees of a perfluorooctanesulphonyl fluoride (POSF) based fluorochemical production facility.

Methods: A retrospective cohort mortality study followed all workers with at least one year of cumulative employment at the facility. The jobs held by cohort members were assigned to one of three exposure subgroups; high exposed, low exposed, and non-exposed, based on biological monitoring data for perfluorooctane sulphonate (PFOS).

Results: A total of 145 deaths were identified in the 2083 cohort members. Sixty five deaths occurred among workers ever employed in high exposed jobs. The overall mortality rates for the cohort and the exposure subcohorts were lower than expected in the general population. Two deaths from liver cancer were observed in the workers with at least one year of high or low exposure (standardised mortality ratio (SMR) $3.08,95 \% \mathrm{Cl} 0.37$ to 11.10 ). The risk of death from bladder cancer was increased for the entire cohort (three observed, SMR 4.81, 95\% Cl 0.99 to 14.06). All three bladder cancers occurred among workers who held a high exposure job (SMR 12.77, 95\% Cl 2.63 to 37.35). The bladder cancer cases primarily worked in non-production jobs, including maintenance and incinerator and wastewater treatment plant operations.

Conclusion: Workers employed in high exposure jobs had an increased number of deaths from bladder cancer; however it is not clear whether these three cases can be attributed to fluorochemical exposure, an unknown bladder carcinogen encountered during the course of maintenance work, and/or nonoccupational exposures. With only three observed cases the possibility of a chance finding cannot be ruled out.
\end{abstract}

$\mathrm{F}$ luorinated organic compounds have been used for decades in a wide variety of industrial and commercial applications, such as specialty lubricants, semiconductor manufacturing, protective barriers or coatings, surfactants, fire retardants, and non-conductive coolants. Perfluorooctanesulphonyl fluoride (POSF, $\mathrm{C}_{8} \mathrm{~F}_{17} \mathrm{SO}_{2} \mathrm{~F}$ ) is the precursor for one line of these specialty chemicals. Using POSF as the basic building block, unique chemistries are created by derivatising POSF through the sulphonyl moiety using conventional hydrocarbon reactions to make amides, oxazolidones, silanes, carboxylates, and alkoxylates as commercial products, most of which are in the form of high molecular weight polymers. The major applications are surface treatments, paper and packaging protectants, and performance chemicals. ${ }^{1}$ Depending on the degree of polymerisation, such POSF based compounds, or their residuals, can degrade or metabolise to perfluorooctanesulphonate (PFOS, $\mathrm{C}_{8} \mathrm{~F}_{17} \mathrm{SO}_{3}{ }^{-}$) to an undetermined degree. The POSF based fluorochemicals have been produced almost exclusively at one facility in the United States and one in Belgium.

Studies of laboratory animals indicate that the liver is the primary target organ for PFOS. ${ }^{1}$ In rats and cynomolgus primates, high doses of PFOS induced enlargement of liver and apparent alterations in metabolic processes, including reduced serum cholesterol levels. ${ }^{2}{ }^{3}$ PFOS was not found to be a developmental toxicant in rats or rabbits. ${ }^{4}$ Higher maternal doses of PFOS increased neonatal mortality, absorptions, resorptions, and reduced weight gain in rat pups. ${ }^{1}$ There were no effects on postnatal neurological development or on fertility and oestrous cycling in offspring in multigeneration studies. Multiple genotoxicity assays indicate PFOS does not present a hazard from interaction with genetic material. ${ }^{1}$ Although the mechanism of toxicity in laboratory animals is not fully understood, it may be due to an effect on fatty acid transport and metabolism, membrane function, peroxisome proliferation, and/or mitochondrial bioenergetics. ${ }^{5-7}$

Recently the presence of PFOS in non-occupationally exposed populations and wildlife, marine mammals, and piscivorous birds raised concerns about the environmental effects of PFOS. ${ }^{189}$ The background levels of PFOS are low and appear to be below the no observable effect level (NOEL) in toxicological studies; however, PFOS is now recognised as a pervasive compound that will persist in the environment and can accumulate in wildlife. These environmental concerns have prompted the phase-out of the production of POSF based chemicals by the major producer (3M Company).

Exposure to higher concentrations of these chemicals occurs in employees of the manufacturing facilities. ${ }^{10} 11$ Medical surveillance of workers has not associated abnormal clinical chemistry results with serum PFOS levels. ${ }^{12} \mathrm{~A}$ mortality study of workers employed at one plant reported an overall lower all cause and cancer cause specific mortality rate than expected, though the population was relatively young (Mandel and Johnson, unpublished report, 1995). Herein we report the results of an update of that cohort mortality study, with specific emphasis on exposures leading to increased serum PFOS levels. This study protocol was

Abbreviations: $\mathrm{Cl}$, confidence interval; ICD, International Classification of Disease; NDI, National Death Index; N-EtFOSE, Nethylperfluorooctane sulphonamide alcohol; PFOA, perfluorooctanoic acid; PFOS, perfluorooctane sulphonate; POSF,

perfluorooctanesulphonyl fluoride; SMR, standardised mortality ratio 
Main messages

- Perfluoroctanesulphonyl fluoride (POSF) based products can be metabolised to perfluorooctane sulphonate (PFOS), to an undetermined degree, which is persistent and pervasive in the environment and can bioaccumulate a variety of species. PFOS, a weak peroxisome proliferator, targets the liver, resulting in hepatic enlargement, and alteration of metabolic processes, most notably a reduction in serum cholesterol.

- The cohort had a standardised mortality ratio (SMR) for bladder cancer that was significantly increased, which was based on three deaths. PFOS, a final metabolite of POSF based chemistry, is not genotoxic, is soluble in urine at the concentrations present in employees, and failed to produce urinary tract tumours or inflammation in two year rat studies and a six month primate study.

- There were no significant associations between employment at the fluorochemical manufacturing plant and mortality from liver disease (cirrhosis or cancer), although the number of observed and expected deaths were few.

- Other occupational exposures and personal habits were not examined in this study.

reviewed and approved by the University of Minnesota Human Subjects Research Committee.

\section{METHODS}

\section{Study population}

The study population worked at a manufacturing facility in Decatur, Alabama, that began production in 1961 and consists of two plants: specialty film (film plant) and specialty materials (chemical plant). The film plant is located approximately 300 yards west of the chemical plant and produces a variety of polyester and non-polyester films. The three major product groups (referred to as focus factories) in the chemical plant are protective chemicals, performance chemicals, and fluoroelastomers. There are more than 700 different processes, with more than $90 \%$ being batch processes. Raw materials and intermediates for each product group may flow through many different production buildings before packaging and shipment. Perfluorooctanesulphonyl fluoride (POSF, $\mathrm{C}_{8} \mathrm{~F}_{17} \mathrm{SO}_{2} \mathrm{~F}$ ) is the major sulphonated fluorochemical manufactured, and is produced via an electrochemical cell process. Biological monitoring (serum measurement) of PFOS has been used to assess the employees' exposure experience to POSF based fluorochemicals at this plant..$^{11}$

Employees may be exposed by one or all routesinhalation, skin contact/absorption, and ingestion-to these POSF based fluorochemical materials. The primary route of exposure may be different for each employee and depends on several different factors, such as process conditions, job tasks, work location, personal hygiene, personal habits, and general work practices. Although POSF based chemicals are the primary fluorochemicals produced at this plant, exposure to other fluorochemicals, including perfluorohexanesulphonyl fluoride and perfluoroctanoate (PFOA), and exposure to nonfluorochemicals, are likely.

\section{Cohort enumeration}

The cohort for this study was enumerated from work history records obtained from the plant. The records of all employees

\section{Policy implications}

- The company that produces these products announced in May 2000 that it was phasing out of the production of POSF based materials because of the persistence and pervasiveness of POSF in the environment.

were abstracted into an electronic database, recording the worker's name, social security number, employee identification number, date of birth, and the dates of any entry on the work history record, including layoffs and leaves of absence, the job department codes, and job title. To be eligible for inclusion in the cohort, a worker had to accrue at least 365 days of cumulative employment at the site by 31 December 1997. Periods of absence due to illness, military leave, maternity leave, or layoff did not contribute to eligibility. Currently employed workers were assigned 31 December 1997 as their last date of employment.

The newly enumerated cohort was linked to records from the original cohort to update employment information, and to verify names, social security numbers, dates of birth, and dates of death for previously identified decedents. Discrepancies identified in the records were resolved through credit reporting agencies, and the Social Security Administration service for epidemiological research studies. The latter reports the most recent account activity of an individual and whether they are recorded as deceased in the Social Security Death Index.

\section{Follow up and determination of vital status}

Eligible cohort members were followed from the day they accrued 365 days of cumulative employment until 31 December 1998 or their date of death. Vital records searches were performed for all eligible cohort members for whom a death certificate was not obtained in the original study. Determination of vital status was made by searching the National Death Index (NDI) for all workers in the original study and new workers included in the cohort. The Social Security Administration data and/or the Social Security Death Index (SSDI) were searched to verify the vital status of workers who terminated employment before 1979 .

The records of cohort members identified as deceased through the NDI or SSDI were manually reviewed to ensure a valid match, and a copy of the death certificate was requested from the state of record. The death certificates were coded by a licensed nosologist using the rules for the International Classification of Disease (ICD) version in effect at the time of death, which allowed the use of actual (unadjusted) mortality reference data. Decedents identified through NDI for which a death certificate could not be obtained, or if the cause of death could not be coded, were included in the analysis in the "other" cause of death category.

\section{Exposure assessment}

The relative differences in serum PFOS by job were determined by a comprehensive assessment conducted in 1998. ${ }^{10}$ In this study, a total of 232 employees were randomly selected for serum sampling with $80 \%$ participation $(\mathrm{n}=126$ chemical plant; $60=$ film plant $)$. Serum samples were extracted using an ion pairing extraction procedure and were quantitatively analysed for serum PFOS using high pressure liquid chromatography/electrospray tandem mass spectrometry methods. The geometric mean serum PFOS level for chemical plant employees was 0.9 ppm (95\% CI 0.8 to 1.1); for film plant employees it was $0.1 \mathrm{ppm}$ (95\% CI 0.1 to 0.1$)$. The majority of film plant jobs have no direct workplace exposure to fluorochemicals. Their serum levels 
are thought to be due, to a large extent, to environmental exposure in proximity to the chemical plant. Chemical plant jobs were classified into eight categories: cell operators, chemical operators, maintenance workers (primarily mechanics and electricians), mill operators, waste treatment plant operators, engineers/laboratory workers, supervisors/ managers, and administrative assistants. The highest geometric mean level of serum PFOS was observed in cell operators $(2.0 \mathrm{ppm})$, followed by the waste operators (1.5 ppm), chemical operators (1.5 ppm), and maintenance workers (1.3 ppm). Supervisors/managers $(0.9 \mathrm{ppm})$, mill operators $(0.6 \mathrm{ppm})$, engineer/lab workers $(0.4 \mathrm{ppm})$, and administrative assistants $(0.4 \mathrm{ppm})$ had lower geometric mean serum PFOS levels. Mill operators are often entry level positions that usually progress to chemical operators within a few years of hire.

As mentioned previously, exposure to other fluorochemicals, including PFOA, are possible. Until 1998, PFOA was not manufactured at this facility. Rather, PFOA exposures were the result of it being produced as a by-product of the electrolytic cell production or in its use as an emulsifier in fluoropolymer production. Although observed at slightly lower levels, the serum PFOA levels correlated with their serum PFOS levels. ${ }^{10}$

Because production processes have remained constant over time, a simple exposure matrix was developed based on the work history records of the study cohort. With the knowledge of the major job specific serum PFOS levels, a company industrial hygienist and epidemiologist assigned each unique job and department combination in the work history records to one of the following three major exposure categories:

- No workplace exposure to POSF based fluorochemicals (encompasses film plant jobs).

- Low potential workplace exposure to POSF based fluorochemicals (includes such jobs as engineers, quality control technicians, environmental, health, and safety workers, administrative assistants, and managers).

- High potential workplace exposure to POSF based fluorochemicals (includes cell operators, chemical operators, maintenance workers, mill operators, waste operators, and crew supervisors).

Hereafter these three categories will be referred to as the nonexposed, low exposed, and high exposed subcohorts, respectively. All exposure assignments were made without knowledge of the mortality outcomes. An additional classification for cumulative exposure assigned the non-exposed, low exposure, and high exposure jobs relative POSF based job exposure value of 1,3 , and 10 , respectively, based on biomonitoring data. The years spent in each job were multiplied by the relative weights to develop a quantitative exposure metric for examining dose-response relations.

\section{Analysis}

The mortality experience of the cohort was compared to that of the state of Alabama. An additional reference population using 23 regional counties was also used to check the results and rule out large variations within the state. The reference data, which are derived from National Center for Health Statistics data, were obtained from the Mortality Population Data System (MPDS) centre at the University of Pittsburgh. These reference data are age (five year), gender, race, and calendar period (five year) specific, and are coded using the rules for the ICD version in effect for the calendar period.

Age, gender, and calendar period adjusted standardised mortality ratios (SMR) were computed for all cause and specific causes of death using the Alabama reference data. The SMRs and 95\% confidence intervals were computed using standard life table methods with the PC Life Table Analysis System (PCLTAS) software developed by the National Institutes of Occupational Safety and Health (NIOSH). ${ }^{13}$ The race of the workers was not available from the company records, but the population of plant employees is predominantly Caucasian; therefore the reference rates for Caucasians were used for this analysis.

The all cause and cause specific SMRs were computed for the entire cohort and the subcohorts of ever high exposure, ever low exposure, and non-exposed. This initial summary analysis was followed by an exposure specific analysis in which the exposed subcohorts were restricted to workers who had at least one year of cumulative employment in jobs with high or low exposure. For this analysis the period of follow up began when the individual reached one year of cumulative exposure, thereby counting person-time and deaths that occurred after the minimum exposure was accrued.

Causes of death potentially related to fluorochemical exposure were analysed by duration of employment in the three exposure subgroups. The causes of death of a priori interest were cancers of the digestive system, in particular liver cancer and liver cirrhosis, because in animals, PFOS circulates through the enterohepatic circulation and concentrates in the liver. ${ }^{14}$ Other causes of death that appeared to be in excess in one or more of the fluorochemical exposed groups were also evaluated by duration of exposure.

\section{RESULTS}

Of the 3512 workers identified, a total of 2083 workers met the one year enrolment criteria. Of these, 47\% (982) worked at some time in jobs where exposure to POSF based fluorochemicals was considered high (table 1). Approximately 14\% (289) worked in low exposure areas, but never held a job in the high exposure areas, and 812 $(39 \%)$ were considered to have no or minimal workplace exposure to fluorochemicals. The high exposure group was modestly younger than the other workers, but had a longer average tenure at the plant than the remainder of the cohort. Male workers made up $83 \%$ of the cohort and $84 \%$ of the high exposure subcohort. There were 145 deaths identified in the cohort, with 65 deaths in the high exposure group, 27 in the low exposure group, and 53 in the non-exposed group. Death certificates were obtained and coded for 139 (96\%) of the decedents. The six missing death certificates were in the high exposed group $(n=3)$, the low exposure group $(n=2)$, and the non-exposed group $(\mathrm{n}=1)$. The cohort accrued 50972 person-years of follow up.

There were 782 workers who worked a minimum of one year in high exposure jobs and 1065 workers who worked a minimum of one year in high or low exposure jobs. The latter subcohort included workers who held high and low exposure jobs for less than one year, but had a combined exposure period of one year. Fifty three deaths were among those working one year or more in high exposure jobs, and an additional 29 deaths were in the combined high and low exposure group.

The all cause and cause specific mortality rates for the entire cohort were lower than expected compared to the general population of Alabama; 145 observed and 230 expected (SMR 0.63, 95\% CI 0.53 to 0.74) (table 2). A similar pattern was observed for all deaths from cancer; 39 observed, 54 expected (SMR $0.72,95 \%$ CI 0.51 to 0.98 ). The all cause and all cancer causes of death were fewer than expected for the high exposure (table 3 ), low exposure (table 4), and nonexposed (table 5) subcohorts. When restricted to workers who accrued at least one year of employment in the high exposure (table 6), and high or low exposure subgroups (data not shown), the standardised mortality ratios for all causes of death and all malignant neoplasms were well below unity. 
Table 1 Characteristics of eligible cohort members by PFOS exposure category

\begin{tabular}{|c|c|c|c|c|}
\hline & High exposed* & Low exposed $\dagger$ & Non-exposed $\ddagger$ & All \\
\hline Total & 982 & 289 & 812 & 2083 \\
\hline \multicolumn{5}{|l|}{ Gender } \\
\hline $\begin{array}{l}M \\
(\%)\end{array}$ & $\begin{array}{l}826 \\
(84)\end{array}$ & $\begin{array}{l}204 \\
(71)\end{array}$ & $\begin{array}{l}700 \\
(86)\end{array}$ & $\begin{array}{l}1730 \\
(83)\end{array}$ \\
\hline $\mathrm{F}$ & 156 & 85 & 112 & 353 \\
\hline (\%) & (16) & (29) & (14) & (17) \\
\hline \multicolumn{5}{|l|}{ Age at follow up } \\
\hline Median & 49.7 & 52.9 & 51.4 & 50.9 \\
\hline Interquartile range & $43.9-56.8$ & $46.1-60.2$ & $46.4-56.9$ & $45.1-57.1$ \\
\hline \multicolumn{5}{|l|}{ Year of birth } \\
\hline Median & 1948 & 1945 & 1947 & 1947 \\
\hline Interquartile range & $1941-54$ & $1937-52$ & $1941-52$ & $1941-53$ \\
\hline \multicolumn{5}{|l|}{ Years of employment } \\
\hline Median & 16.7 & 10.4 & 9.9 & 13.2 \\
\hline Interquartile range & $4.5-26.1$ & $2.7-22.7$ & $2.7-25.2$ & $3.4-25.3$ \\
\hline \multicolumn{5}{|c|}{ Person-years of follow up } \\
\hline Median & 24.8 & 26.3 & 26.7 & 25.9 \\
\hline Interquartile range & $19.4-31.1$ & $20.8-31.9$ & $21.4-30.1$ & $20.4-30.8$ \\
\hline Deaths & 65 & & 53 & 145 \\
\hline
\end{tabular}

*Ever employed in high exposure job.

†Ever employed in low exposure jobs, but never a high exposure job.

$\ddagger$ No or minimal workplace fluorochemical exposure.

Five deaths from cirrhosis of liver were identified in the entire cohort, two of which occurred in the high exposure group; however this did not exceed the number expected (2.5). Two deaths from liver cancer were observed in the entire cohort, with 1.24 expected (SMR 1.61, 95\% CI 0.20 to 5.82). One liver cancer case was observed in the subcohort employed in a high exposure jobs ( 0.4 expected, table 6$)$, and the other held a low exposure job for at least one year. The SMR for liver cancer among workers who held high or low exposure jobs for at least one year was 3.08 (95\% CI 0.37 to 11.10). The worker employed in the high exposure job worked at the facility for just over one year. The worker employed in the lower exposure jobs worked for approximately 10 years before retiring. Medical validation by followback to the physician of record on the death certificate was conducted for the deaths from liver cancer. Both cases were confirmed to be primary hepatocellular carcinomas.

Three deaths were attributed to malignant neoplasms of the bladder (0.62 expected in the entire cohort, SMR 4.81, $95 \%$ CI to 0.99 to 14.05 ). The workers who died from bladder cancer were in the sub cohort that worked in jobs with high exposure for at least one year (0.19 expected, SMR 16.12, 95\% CI 3.32 to 47.41 , table 6). All three cases of bladder cancer were male and each had worked in high exposure jobs for at least five years; the SMR for five or more years of employment in a high exposure job was 25.5 ( 3 observed, 0.12 expected). With only three cases of bladder cancer, all with experience in high exposure jobs, an exposure response analysis using the weighted cumulative exposure metric was not informative. The results for bladder cancer in relation to

\begin{tabular}{|c|c|c|c|c|}
\hline Cause* $^{*}$ & Observed & Expected & SMR & $95 \% \mathrm{Cl}$ \\
\hline All deaths & 145 & 230.09 & 0.63 & 0.53 to 0.74 \\
\hline \multicolumn{5}{|l|}{ Cancers } \\
\hline All malignant neoplasms & 39 & 54.26 & 0.72 & 0.51 to 0.98 \\
\hline Digestive organs and peritoneum & 5 & 9.78 & 0.51 & 0.17 to 1.19 \\
\hline Oesophagus & 2 & 1.15 & 1.76 & 0.21 to 6.35 \\
\hline Large intestine & 1 & 3.35 & 0.30 & 0.01 to 1.66 \\
\hline Biliary passages and liver & 2 & 1.24 & 1.61 & 0.20 to 5.82 \\
\hline Respiratory system & 15 & 21.01 & 0.71 & 0.40 to 1.18 \\
\hline Bronchus, trachea, lung & 15 & 20.33 & 0.74 & 0.41 to 1.22 \\
\hline Breast & 2 & 1.28 & 1.57 & 0.19 to 5.66 \\
\hline Urinary organs & 3 & 1.89 & 1.59 & 0.33 to 4.65 \\
\hline Bladder and other urinary & 3 & 0.62 & 4.81 & 0.99 to 14.06 \\
\hline Malignant melanoma & 3 & 1.80 & 1.67 & 0.34 to 4.88 \\
\hline Lymphatic and haematopoietic & 4 & 5.68 & 0.70 & 0.19 to 1.80 \\
\hline \multicolumn{5}{|l|}{ Non-malignant causes } \\
\hline Cerebrovascular disease & 5 & 7.13 & 0.70 & 0.23 to 1.64 \\
\hline All heart disease & 35 & 62.53 & 0.56 & 0.39 to 0.78 \\
\hline Non-malignant respiratory disease & 1 & 10.6 & 0.09 & 0.00 to 0.52 \\
\hline Cirrhosis of liver & 5 & 5.89 & 0.85 & 0.27 to 1.98 \\
\hline All accidents & 27 & 36.65 & 0.74 & 0.49 to 1.07 \\
\hline Motor vehicle accidents & 15 & 22.05 & 0.68 & 0.38 to 1.12 \\
\hline All other accidents & 12 & 14.61 & 0.82 & 0.42 to 1.44 \\
\hline Violence & 10 & 19.27 & 0.52 & 0.25 to 0.95 \\
\hline Suicides & 5 & 11.42 & 0.44 & 0.14 to 1.02 \\
\hline Homicides & 5 & 7.85 & 0.64 & 0.21 to 1.49 \\
\hline
\end{tabular}


Table 3 Cause specific deaths and standardised mortality ratios for all causes and selected causes of death for cohort members ever employed in a high exposure job

\begin{tabular}{lcccc}
\hline Cause* & Observed & Expected & SMR & 95\% Cl \\
\hline All deaths & 65 & 93.56 & 0.69 & 0.54 to 0.89 \\
Cancers & 18 & & & \\
$\quad$ All malignant neoplasms & 2 & 21.54 & 0.84 & 0.50 to 1.32 \\
Digestive organs and peritoneum & 1 & 3.91 & 0.51 & 0.06 to 1.85 \\
Oesophagus & 1 & 0.46 & 2.16 & 0.05 to 12.02 \\
Biliary passages and liver & 7 & 0.50 & 2.00 & 0.05 to 11.10 \\
Respiratory system & 7 & 8.24 & 0.85 & 0.34 to 1.75 \\
Bronchus, trachea, lung & 3 & 7.97 & 0.88 & 0.35 to 1.81 \\
Urinary organs & 3 & 0.75 & 4.02 & 0.83 to 11.75 \\
Bladder and other urinary organs & 2 & 0.23 & 12.77 & 2.63 to 37.35 \\
Malignant melanoma & 1 & 0.76 & 2.62 & 0.32 to 9.46 \\
Lymphatic and haematopoietic & & 2.31 & 0.43 & 0.01 to 2.40 \\
Non-malignant causes & 2 & & & \\
Cerebrovascular disease & 14 & 2.76 & 0.72 & 0.09 to 2.62 \\
All heart disease & 2 & 2.46 & 0.56 & 0.31 to 0.95 \\
Cirrhosis of liver & 17 & 15.87 & 0.81 & 0.10 to 2.94 \\
All accidents & 9 & 9.60 & 0.94 & 0.62 to 1.72 \\
Motor vehicle accidents & 8 & 6.26 & 1.28 & 0.53 to 1.78 \\
All other accidents & 3 & 8.58 & 0.36 & 0.07 to 1.05 \\
Violence & 1 & 4.97 & 0.20 & 0.01 to 1.12 \\
Suicides & 2 & 3.42 & 0.59 & 0.07 to 2.11 \\
Homicides & & & & \\
\hline * Cause not listed if not observed. & & & & \\
\hline
\end{tabular}

fluorochemical exposure did not change when the reference population was the local counties rather than the entire state of Alabama. The job classifications of the workers who died from bladder cancer were classified as high exposure; however they were not exclusively chemical production jobs. The majority of their work history entailed work in maintenance work or in the plant incinerator or wastewater treatment plant.

\section{DISCUSSION}

This study evaluated the mortality experience of workers with at least one year of employment at a facility that produced perfluorooctanesulphonyl fluoride based fluorochemicals. Exposures to these chemicals are of particular interest because an end stage metabolite, PFOS, has been shown to be pervasive and biopersistent, leading to bioaccumulation in human and non-human species. ${ }^{189}$ The overall mortality rates were below those expected for most causes. An excess of death from bladder cancer was detected and limited to workers who held high POSF exposure jobs for at least five years. Other causes of death were below the number expected or occurred too infrequently to evaluate with confidence.

Some limitations must be considered when interpreting the results of this mortality analysis. Although several methods of follow up were employed to identify decedents in this cohort, the possibility remains that some deaths were not ascertained. A death certificate was not obtained for six known decedents; thus they were not included in the cause specific death analysis. The extent to which these limitations would affect the results is unknown, but would have the largest effect on the analysis if the unknown and uncoded causes of death were attributed to relatively rare causes of death, including liver, bladder, kidney, and prostate cancer.

Another limitation in this study is the lack of employee specific exposure data for PFOS and other fluorochemicals. Employment records were used to classify the exposure status of cohort members. The relative exposure estimates were guided with biomonitoring data from a previous exposure assessment, which reduced misclassification, but did not eliminate it. Information of potentially confounding factors, for example, smoking, were not available for this

Table 4 Cause specific deaths and standardised mortality ratios for all causes and selected causes of death for cohort members ever employed in a low exposure job, but never a high exposure job

\begin{tabular}{lllll}
\hline Cause* & Observed & Expected & SMR & 95\% Cl \\
\hline All deaths & 27 & 43.43 & 0.64 & 0.42 to 0.93 \\
Cancers & & & & \\
$\quad$ All malignant neoplasms & 6 & 11.47 & 0.52 & 0.19 to 1.14 \\
$\quad$ Digestive organs and peritoneum & 2 & 2.02 & 0.99 & 0.12 to 3.57 \\
$\quad$ Large intestine & 1 & 0.70 & 1.43 & 0.04 to 7.94 \\
$\quad$ Biliary passages and liver & 1 & 0.25 & 3.94 & 0.10 to 21.88 \\
$\quad$ Respiratory system & 4 & 4.60 & 0.87 & 0.24 to 2.22 \\
$\quad$ Bronchus, trachea, lung & 4 & 4.46 & 0.90 & 0.24 to 2.29 \\
Non-malignant causes & 2 & & & \\
$\quad$ Cerebrovascular disease & 7 & 1.61 & 1.25 & 0.15 to 4.50 \\
$\quad$ All heart disease & 1 & 0.93 & 0.54 & 0.22 to 1.12 \\
$\quad$ Cirrhosis of liver & 1 & 4.33 & 0.23 & 0.01 to 5.95 \\
$\quad$ All accidents & 3 & 2.34 & 1.28 & 0.26 to 3.74 \\
$\quad$ Violence & 1 & 1.45 & 0.69 & 0.02 to 3.83 \\
Suicides & 2 & 0.89 & 2.24 & 0.27 to 8.08 \\
$\quad$ Homicides & & & & \\
\hline *Cause not listed if not observed. & & & & \\
\hline
\end{tabular}


Table 5 Cause specific deaths and standardised mortality ratios for all causes and selected causes of death for cohort members who only worked in non-exposed jobs

\begin{tabular}{lllll}
\hline Cause & Observed & Expected & SMR & $95 \% \mathrm{Cl}$ \\
\hline All deaths & 53 & 88.31 & 0.60 & 0.45 to 0.79 \\
Cancers & 15 & 20.45 & 0.73 & 0.41 to 1.21 \\
$\quad$ All malignant neoplasms & 1 & 3.72 & 0.27 & 0.01 to 1.49 \\
$\quad$ Digestive organs and peritoneum & 1 & 0.44 & 2.25 & 0.06 to 12.51 \\
Oesophagus & 4 & 7.90 & 0.51 & 0.14 to 1.30 \\
Respiratory system & 4 & 7.64 & 0.52 & 0.14 to 1.34 \\
Bronchus, trachea, lung & 2 & 0.39 & 5.11 & 0.62 to 18.45 \\
Breast & 1 & 0.72 & 1.38 & 0.03 to 7.67 \\
Malignant melanoma & 3 & 2.19 & 1.37 & 0.28 to 4.00 \\
Lymphatic and haematopoietic & 1 & & & \\
Non-malignant causes & 14 & 23.72 & 0.38 & 0.01 to 2.12 \\
$\quad$ Cerebrovascular disease & 1 & 3.81 & 0.26 & 0.32 to 0.99 \\
$\quad$ All heart disease & 2 & 2.37 & 0.84 & 0.10 to 1.46 \\
$\quad$ Non-malignant respiratory disease & 9 & 14.67 & 0.61 & 0.28 to 1.17 \\
$\quad$ Cirrhosis of liver & 6 & 8.82 & 0.68 & 0.25 to 1.48 \\
$\quad$ All accidents & 3 & 5.84 & 0.51 & 0.11 to 1.50 \\
Motor vehicle accidents & 4 & 7.79 & 0.51 & 0.14 to 1.31 \\
$\quad$ All other accidents & 3 & 4.59 & 0.65 & 0.13 to 1.91 \\
$\quad$ Violence & 1 & 3.20 & 0.31 & 0.01 to 1.74 \\
$\quad$ Suicides & & & & \\
$\quad$ Homicides & & & & \\
\hline *Cause not listed if not observed. & & & & \\
\hline
\end{tabular}

cohort. Finally, the focus of this study was fluorochemical exposure, so the influence of other workplace exposures was not assessed.

The number of deaths from bladder cancer was more than expected, based on the prevailing rates for Alabama and the regional counties. In the current analysis three cases were observed; however the fact that all three cases worked for at least five years in high exposure jobs warrants further evaluation. Possible explanations for the excess include: (1) the cancers are due to exposure to PFOS and other fluorochemicals; (2) the cancers are attributable to another, undetermined occupational exposure; (3) the cancers are related to non-occupational factors such as smoking or other personal habits; and (4) chance. With only three cases the possibility that the finding is due to chance cannot entirely be ruled out. However, given the magnitude of the risk estimate it would take many years of additional follow up with no further deaths from bladder cancer for this excess to fully attenuate to null.

Current toxicological evidence does not indicate that the bladder is a target of PFOS. Two year feeding bioassays in rats of N-ethylperfluorooctane sulphonamide alcohol (NEtFOSE) and PFOS have not shown an increased risk of bladder tumours. ${ }^{1}{ }^{15} 16$ The former compound can metabolise to an undetermined degree to PFOS. Most bladder carcinogens are genotoxic and/or precipitate in the urine. PFOS and related chemistries are neither genotoxic nor insoluble in urine at the levels measured in employees. ${ }^{1}$

A known risk factor for bladder cancer is tobacco smoking. ${ }^{17}$ Data on smoking habit were not available for this analysis. However, in this cohort the rates of other smoking related cancers, including lung cancer, were not increased, suggesting that the cohort as a whole did not smoke appreciably more than the general population.

Table 6 Cause specific standardised mortality ratios for workers employed for at least one year in a high exposure job

\begin{tabular}{lcccl}
\hline Cause* & Observed & Expected & SMR & $95 \% \mathrm{Cl}$ \\
\hline All deaths & 53 & 73.26 & 0.72 & 0.54 to 0.95 \\
Cancers & 14 & 16.67 & 0.84 & 0.46 to 1.41 \\
$\quad$ All malignant neoplasms & 2 & 3.05 & 0.66 & 0.08 to 2.37 \\
Digestive organs and peritoneum & 1 & 0.37 & 2.73 & 0.07 to 15.16 \\
Oesophagus & 1 & 0.39 & 2.57 & 0.06 to 14.26 \\
Biliary passages and liver & 6 & 6.45 & 0.93 & 0.34 to 2.03 \\
Respiratory system & 6 & 6.24 & 0.96 & 0.35 to 2.09 \\
Bronchus, trachea, lung & 3 & 0.59 & 5.11 & 1.05 to 14.93 \\
Urinary organs & 3 & 0.19 & 16.12 & 3.32 to 47.14 \\
Bladder and other urinary organs & 1 & 0.60 & 1.67 & 0.04 to 9.25 \\
Malignant melanoma & 1 & 1.80 & 0.56 & 0.01 to 3.08 \\
Lymphatic and haematopoietic & 2 & 2.14 & 0.93 & 0.11 to 3.37 \\
Non-malignant causes & 12 & 19.52 & 0.61 & 0.32 to 1.07 \\
$\quad$ Cerebrovascular disease & 2 & 1.93 & 1.03 & 0.13 to 3.73 \\
All heart disease & 13 & 12.46 & 1.04 & 0.56 to 1.78 \\
Cirrhosis of liver & 6 & 7.53 & 0.80 & 0.29 to 1.74 \\
All accidents & 7 & 4.93 & 1.42 & 0.57 to 2.92 \\
Motor vehicle accidents & 3 & 6.63 & 0.45 & 0.09 to 1.32 \\
All other accidents & 1 & 3.93 & 0.25 & 0.01 to 1.41 \\
Violence & 2 & 2.71 & 0.74 & 0.09 to 2.67 \\
$\quad$ Suicides & & & & \\
Homicides & & & & \\
\hline *Cause not listed if not observed. & & & & \\
\hline
\end{tabular}


In addition to smoking, bladder cancer has been associated with several occupational exposures. Employment in the textile industry in particular has long been associated with risk of bladder cancer due to the use of aniline dyes and other agents. Several chemicals currently or historically used in the textile industry have been classified by the International Agency for Research on Cancer (IARC) as class 1 (carcinogenic), class 2A (probably carcinogenic), and class 2B (possibly carcinogenic). The majority of the chemicals evaluated fall into the $2 \mathrm{~B}$ category, including plasticisers such as acetamide and di(2-ethyl hexyl)-phthalate, flame retardants such as thiourea, tris (2,3-dibromo propyl phosphate), and antimony trioxide. ${ }^{18}$ Occupational exposure to aromatic amines in the textile and other industries are possibly responsible for up to $25 \%$ of bladder cancer in some countries. ${ }^{19}$ Polycyclic aromatic hydrocarbons (PAHs) exposure has also been linked to bladder cancer. ${ }^{20}$ These associations have been reported in the aluminium industry, and occupations where exposure to coal tar pitch is common. Several other occupational exposures that are not industry specific including metal cutting fluids, diesel exhaust, polybrominated biphenyls, and perchloroethylene and other solvents, have been associated, albeit inconsistently, with bladder cancer in some populations. ${ }^{21-27}$

Although the bladder cancer cases in this cohort occurred among workers who held high PFOS exposed jobs, the specific jobs of the cases suggest that other exposures may have been present. The bladder cancer cases worked mostly in maintenance or at the incinerator and wastewater treatment plant. The exposures in these jobs are not limited to those encountered in a specific chemical production process. Maintenance workers may work in many areas of the plant. The wastewater treatment and incinerator workers are on the receiving end of the waste stream, and have the opportunity to encounter a number of chemical exposures.

Following the initial release of these findings to the company and workers, a review of the current and past presence of known bladder carcinogens at this facility was conducted. A list of known or potential bladder carcinogens was developed, based on review of the medical literature. Forty five such compounds were identified. The historic raw material inventory records kept by the company were searched by CAS number to identify any use of these compounds. These records covered all materials purchased for production use. In addition, plant records were reviewed and key personnel were interviewed to ascertain more specific information on chemical use. Of the 45 compounds initially identified, five were recognised as being used at the manufacturing plant site. Four materials were found to have been part of former, inactive processes. These include 4,4 methylene-dianiline, orthotoluidine, benzidine salts, and butyl benzyl phthalate. The use of these materials ended in the 1960s and 1970s. The chemicals were not widely used in the plant; however the available exposure monitoring and use information was very limited. Exposure controls consisted of ventilation controls and employee training for safe handling practices. One material (melamine) is currently in use in an epoxy capsule (non-fluorochemical) product line. Melamine has been used for the past decade in the chemical plant in epoxy capsule manufacturing. Respiratory protection and local exhaust ventilation have been the primary exposure control methods for melamine. Qualitative exposure assessments indicated exposures to melamine were low based on short exposure task durations and exposure control practices. Chloroprene was also used in several manufacturing processes in the chemical plant in the 1960s and 1970s. Chloroprene is considered a possible human carcinogen; however, the evidence for a role in bladder cancer is equivocal. ${ }^{28}$
There were two deaths from liver cancer in the fluorochemical exposed subcohorts; approximately three times that expected. Both cases were verified as primary cancers by medical record follow-back. Though more than expected, these results are difficult to interpret because they are based on only two cases. The case with the highest fluorochemcial exposure held a high PFOS exposed job for only 14 months. The other case occurred in an older man who worked as a general helper in departments with low exposure for 11 years. There were no liver cancer cases among the workers with much longer duration of employment in high exposure jobs, thus a dose-response relation could not be evaluated.

Death from liver cancer and cirrhosis of the liver were a priori outcomes of interest for this study because PFOS is concentrated in the liver, ${ }^{14}$ and several repeat dose studies have consistently shown that the liver is the primary target organ. ${ }^{1}$ Liver tissue response to high doses of PFOS included the enlargement of liver and apparent alterations in metabolic processes with the reduction in serum cholesterol levels observed as the earliest clinical response to PFOS. These effects occurred in cynomolgus primates at serum PFOS levels at $100 \mathrm{ppm}$, whereas production workers serum levels have averaged $1-2 \mathrm{ppm}$, with the highest around $10 \mathrm{ppm} .^{1}$ Multiple genotoxicity assays suggested PFOS does not present a hazard from interaction with genetic material. Two year feeding bioassays of N-EtFOSE and PFOS in rats have shown marginal increases in hepatocellular adenomas at the highest dose in each study. ${ }^{15}{ }^{16} \mathrm{~N}$-EtFOSE and PFOS produced weak peroxisome proliferation, but neither compound was found to be genotoxic. ${ }^{1}$ Thus, the results of the two year bioassay studies were characterised as a nongenotoxic threshold response for liver tumours.

PFOA, unlike PFOS, is a moderate to strong hepatic peroxisome proliferator in the rat..$^{23}$ A two year bioassay of PFOA in the diet of rats caused an increase of hepatic, pancreas, and Leydig cell adenomas. ${ }^{30}{ }^{32}$ There is considerable debate whether peroxisome proliferators that produce liver tumours in rats are carcinogenic to humans under anticipated conditions and levels of human exposure. ${ }^{33}$

Known causes of primary hepatocellular carcinoma in humans include the hepatitis B and C viruses, and aflatoxins produced by Aspergillus flavus and Aspergillus parasiticus, primarily in foodstuffs. ${ }^{34}$ Hepatitis B virus is thought to be responsible for up to $85 \%$ of liver cancers in endemic areas; however the prevalence of infection in the USA is relatively low. Other contributing causes may include cirrhosis due to excessive alcohol consumption. Exposure to high concentrations of vinyl chloride is associated with angiosarcoma of the liver, a very rare cancer. However, there are no clear occupational causes of primary hepatocellular carcinoma. It is possible that that this finding is due to chance.

In summary, an excess occurrence of death from bladder cancer was observed in this population; however, it is not clear whether these cases can be attributed to fluorochemical exposure, an unknown bladder carcinogen encountered during the course of maintenance work, and/or non-occupational exposures. With only three cases observed in the population the possibility of chance cannot be entirely ruled out. The conditions of a priori interest were fewer than expected or occurred too infrequently to evaluate with confidence. The relatively young age and small size of the cohort currently precludes a detailed analysis by exposure, particularly for less common diseases.

\section{ACKNOWLEDGEMENTS}

The authors wish to thank Nancy Pengra, Mark Bollenbeck, and Diane Kampa for assistance with the mortality follow up, data management, and analysis, and Perry Logan for assistance with exposure assessment.

This work was supported by the $3 \mathrm{M}$ Company. 


\section{Authors' affiliations}

B H Alexander, University of Minnesota, School of Public Health, Division of Environmental and Occupational Health, MMC 807 Mayo Building, 420 Delaware St, SE Minneapolis, MN 55409, USA G W Olsen, J M Burris, J H Mandel, 3M Company, Medical Department, Saint Paul, MN, USA

J S Mandel, Emory University, Rollins School of Public Health, Department of Epidemiology, Atlanta, GA, USA

\section{REFERENCES}

$13 \mathrm{M}$ Company. SIDS initial assessment report: perfluorooctane sulphonic acid and its salts: USEPA Public Docket AR-226. Saint Paul: 3M Company, 2000.

2 Seacat AM, Butenhoff JL. Comparative sub-chronic toxicity of perfluorooctane sulphonate (PFOS) and $\mathrm{N}$-ethyl perfluorooctanesulphonamide ethanol (NEtFOSE) in the rat. Toxicologist 2001;60:348.

3 Seacat AM, Thomford PJ, J HK, et al. Subchronic toxicity studies on perfluorooctanesulphate potassium salt in cynomolgus monkeys. Toxicol Sci 2002;68:249-64

4 Case MT, York FG, Christian MS. Rat and rabbit oral developmental toxicology studies with two perfluorinated compounds. Int J Toxicol 2001;20:101-9.

5 Luebker DJ, Hansen KJ, Bass N, et al. Interactions of fluorochemicals with rat liver fatty acid-binding protein. Toxicology 2002;176:175-85.

6 Wallace KB, Luebker DJ, Butenhoff JL, et al. Perfluorooctanesulphonate and 2(n-ethylperfluorooctanesulphonaminde)-ethyl alcohol are peroxisome proliferators in rats, but not guinea pigs. Toxicologist 2001;60:348.

7 Starkov A, Butenhoff JL, Seacat AM, et al. Structural determinants of mitochondrial dysfunction caused by in vitro exposure to selected perfluorootanyl compounds. Toxicologist 2001;60:1658.

8 Hansen KJ, Clemen LA, Ellefson ME, et al. Compound-specific, quantitative characterization of organic fluorochemicals in biological matrices. Environ Sci Technol 2001;35:766-70.

9 Giesy JP, Kannan K. Global distribution of perfluoroctane sulphonate and related perfluorinated compounds in wildlife. Environ Sci Technol 2001;35:1339-42

10 Olsen GW, Logan PW, Simpson CA, et al. An occupational exposure assessment of a perfluorooctanesulfonyl fluoride production site: biomonitoring. Am Indust Hyg Assoc J In press.

11 Olsen GW, Logan PW, Simpson CA, et al. Fluorochemical exposure assessment of Decatur chemical and film plant employees. USEPA Public Docket AR-226. Saint Paul: 3M Company, 1999

12 Olsen GW, Burris JM, Mandel JH, et al. Serum perfluorooctane sulfonate and hepatic and lipid clinical chemistry tests in fluorochemical production employees. J Occup Environ Med 1999:41:799-806.

13 National Institutes for Occupational Safety and Health. PC LTAS: life table analysis system for use on the PC. Cincinnati: US Department of Health and Human Services, 1998.

14 Johnson JD, Gibson SJ, Ober RE. Extent and route of excretion and tissue distribution of total carbon-14 in rats after a single i.v. dose of FC-94- ${ }^{14}-\mathrm{C}$. Project 8900310200. Saint Paul, MN: Riker Laboratories Inc., 1979.
15 Seacat AM, Thomford PJ, Butenhoff JL. Terminal observations in SpragueDawley rats after lifetime dietary exposure to potassium perfluorooctane sulfonate. Toxicologist 2002;66:185.

16 Thomford PJ, Seacat AM, Butenhoff JL. Terminal observations in SpragueDawley rats after liftime dietary exposure to $\mathrm{N}$-ethyl perfluorooctanesulfonamide ethanol. Toxicologist 2002;66:185

17 Silverman DT, Morrison AS, Devesa SS. Bladder cancer. In: Schottenfeld D Fraumeni JF, eds. Cancer epidemiology and prevention. New York: Oxford University Press, 1996:1156-79.

18 International Agency for Research on Cancer. IARC monographs on the evaluation of carcinogenic risks to humans: Some flame retardants and textile chemicals and exposures in the textile manufacturing industry. Lyon: IARC, 1990.

19 Vineis P, Pirastu R. Aromatic amines and cancer. Cancer Causes Control 1997:8:346-55.

20 Boffetta P, Jourenkova N, Gustavsson P. Cancer risk from occupational and environmental exposure to polycyclic aromatic hydrocarbons. Cancer Causes Control 1997:8:444-72.

21 Ward EM, Burnett CA, Ruder A, et al. Industries and cancer. Cancer Causes Control 1997;8:356-70.

22 Bonassi S, Merlo F, Pearce N, et al. Bladder cancer and occupational exposure to polycyclic aromatic hydrocarbons. Int J Cancer 1989;44:648-51.

23 Hours M, Dananche B, Fevotte J, et al. Bladder cancer and occupational exposures. Scand J Work Environ Health 1994;20:322-30

24 Steineck G, Plato N, Norell SE, et al. Urothelial cancer and some industryrelated chemicals: an evaluation of the epidemiologic literature. Am J Ind Med 1990;17:371-91.

25 Anton-Culver $\mathbf{H}$, Lee-Feldstein A, Taylor TH. Occupation and bladder cancer risk. Am J Epidemiol 1992;136:89-94.

26 Weiss NS. Cancer in relation to occupational exposure to perchloroethylene. Cancer Causes Control 1995;6:257-66.

27 Porru S, Aulenti V, Donato F, et al. Bladder cancer and occupation: a casecontrol study in northern Italy. Occup Environ Med 1996:53:6-10.

28 International Agency for Research on Cancer. IARC monographs on the evaluation of carcinogenic risks to humans: Re-evaluation of some organic chemicals, hydrazine and hydrogen peroxide (Part One). Lyon: IARC, 1999.

29 Haughom B, Spydevold O. The mechanism underlying the hypolipemic effect of perfluorooctanoic acid, perfluorooctane sulphonic acid (PFOSA) and clofibric acid. Biochim Biophys Acta 1992;1128:65-72.

30 Sibinski L. Two-year oral (diet) toxicity/carcinogenicity of fluorochemical FC143 in rats. Saint Paul: Riker Laboratories, 1987.

31 Biegel LB, Liu RCM, Hurtt ME, et al. Effects of ammonium perfluorooctanoate on Leydig cell function: in vitro, in vivo, and ex vivo studies. Toxicol Appl Pharmacol 1995; 134:18-25.

32 Obourn JD, Frame SR, Bell RH, et al. Mechanisms for the pancreaeatic oncogenic effects of peroxisome proliferator Wyeth-14,643. Toxicol Appl Pharmacol 1997:145.

33 Cattley RC, Deluca J, Elcanbe E, et al. Do peroxisome proliferating compunds pose a hepatocarciongenic hazard to humans. Regul Toxicol Pharmacol 1998;27:47-60

34 London WT, McGlynn KA. Liver cancer. In: Schottenfeld D, Fraumeni JF, eds. Cancer epidemiology and prevention. New York: Oxford University Press, 1996:772-93 